\title{
Overcoming Diagnostic Dilemma and Optimizing Intervention with Optical Coherence Tomographic Guidance in an Angiographically Ambiguous Renal Artery Stenosis Due to Fibromuscular Dysplasia
}

\author{
Parminder Singh Otaal (D) \\ Akash Batta (iD) \\ Saroj Kumar Sahoo \\ Rajesh Vijayvergiya
}

Department of Cardiology, Advanced Cardiac Centre, Post Graduate Institute of Medical Education and Research (PGIMER), Chandigarh, India
Correspondence: Parminder Singh Otaal Department of Cardiology, Advanced Cardiac Centre, Post Graduate Institute of Medical Education and Research, Sector 12, Chandigarh, 160 012, India Tel +919814526747

Email psotaal@gmail.com

\begin{abstract}
With the advent of endovascular therapy, conventional angiography has replaced histology as a diagnostic gold standard for fibromuscular dysplasia (FMD). However, angiographic findings are not precisely diagnostic in isolated focal renal artery stenosis (RAS) in FMD. Optical coherence tomography (OCT) imaging by reflecting in vivo histology may improve the diagnostic accuracy in such cases. Restenosis following balloon angioplasty of RAS in FMD occurs frequently and often requires optimized reintervention. However, the role of OCT in the diagnostic evaluation of focal RAS in FMD and in optimizing the intervention is not well studied. We present a young female with resistant secondary hypertension due to bilateral focal RAS on angiography. In the absence of an angiographic string of beads appearance, the diagnoses remained uncertain. However, OCT imaging diagnosed a multifocal disease with characteristic changes of fibro-medial dysplasia, akin to its histological description in the literature. The patient underwent bilateral balloon angioplasty with good angiographic results. After one year on follow-up, relapse of uncontrolled systemic hypertension occurred due to bilateral restenosis. Despite a satisfactory repeat balloon angioplasty of the left-sided re-stenotic lesion, a significant residual pressure gradient persisted. Usage of OCT guided oversized balloon and stent safely achieved good results with complete resolution of the pressure gradient. Right renal artery stenosis required balloon angioplasty alone. She had complete resolution of hypertension without any medications at six months of follow-up. In conclusion, OCT can supplement other diagnostic modalities in providing a proper diagnosis in an angiographically ambiguous focal RAS in FMD. A persistent residual pressure gradient across the lesion despite good angiographic results may be optimized with OCT guidance during the intervention.
\end{abstract}

Keywords: fibromuscular dysplasia, focal renal artery stenosis, optical coherence tomography imaging, diagnosis, restenosis, stenting

\section{Introduction}

Fibromuscular dysplasia (FMD) is an uncommon cause of secondary hypertension due to associated renal artery stenosis (RAS), which usually is bilateral. ${ }^{1}$ Although angiography is considered a gold standard for diagnosis and classification of FMD but has some limitations in the characterization of vessel wall and classification of disease, which can be overcome with Optical Coherence Tomography (OCT). ${ }^{2}$ When associated with resistant hypertension, renal angioplasty is indicated. ${ }^{1,3}$ 
However, in contrast to atherosclerotic disease, balloon angioplasty rather than stenting is a preferred strategy. ${ }^{1,3,4}$ Outcome following revascularization is variable, and on average, only $36 \%$ achieve cure of hypertension. ${ }^{5}$ Younger age and shorter duration of hypertension correlate with increased likelihood of resolution of hypertension. ${ }^{1,3,6}$ Focal disease has been shown to fare better in terms of outcome than the multifocal disease. Finally, the rate of reintervention after balloon angioplasty remains high, around $28 \%$ at five years. ${ }^{3,6}$

We report a young female with resistant hypertension secondary to bilateral RAS due to FMD, where OCT characterization helped in the diagnosis and proper classification of an angiographically ambiguous focal stenosis. She underwent successful OCT guided balloon angioplasty to bilateral renal arteries with reasonable control of her blood pressure. However, one year after the intervention, she had a relapse of uncontrolled hypertension due to bilateral restenosis requiring re-intervention. OCT guidance during re-intervention encouraged the use of an oversized stent for residual translesional pressure gradient with excellent angiographic, tomographic and clinical outcome.

\section{Case Presentation}

History dates back to 3 years back when she was detected to be hypertensive during pregnancy and prescribed labetalol, amlodipine and hydrochlorothiazide. One and half years later, at the age of 30 years, she presented to us with complaints of dyspnea and headache for last six months. She also has a history of hospitalization for pulmonary edema 15 days before that presentation. Her clinical examination was inconspicuous except for high blood pressure of $210 / 120 \mathrm{mmHg}$. In addition to previous medications, clonidine and prazosin were added to control her blood pressure.

Her biochemical profile, including serum creatinine $(0.8 \mathrm{mg} / \mathrm{dL})$ and estimated Glomerular Filtration Rate (103 mL/min), was normal. Abdominal ultrasound showed bilateral normal-sized kidneys with preserved corticomedullary differentiation. Electrocardiogram was suggestive of left ventricular hypertrophy, and echocardiography revealed a left ventricular ejection fraction (LVEF) of $45 \%$. Renal artery Doppler suspected bilateral renal artery stenosis (RAS). Contrast-enhanced Computed Tomography (CT) (Figure 1A) and conventional angiography (Figure 1B and C) showed critical focal stenosis in the mid-segment of the bilateral main renal arteries, which in a young female, although suggestive but is not diagnostic of FMD. No string of beads appearance typical of FMD was observed.

To help diagnose and rule out other differentials, Optical Coherence Tomography (OCT) imaging was performed before intervention by the frequency-domain system (ILUMIEN TM Optis System using Dragonfly Optis Catheter TM St. Jude Medical, MA, USA). This is a US FDA approved modality for use in peripheral arteries. OCT imaging of the left renal artery revealed a characteristic string of beads appearance in longitudinal reconstruction (Figure 1D). Cross-sectional images show an intima-media dissection (Figure 1E) and homogeneously thickened media as a low backscatter layer with a high backscatter in the inner half suggestive of fibrosis. These features are characteristic of predominantly medial fibroplasia type of FMD. OCT quantified luminal area stenosis to $92 \%$ (Figure $1 \mathrm{~F}$ ) with a normal reference segment (Figure 1G). OCT of the right renal artery (done after balloon) angioplasty revealed a homogeneously thickened media with minimal fibrosis suggestive of medial fibroplasia (Figure 1H).

Due to resistant hypertension, she was offered renal angioplasty, which she accepted. As OCT measured distal reference diameter was $4.1 \mathrm{~mm}$, the left and right RAS was dilated with a $4 \mathrm{mmx} 12 \mathrm{~mm}$ semi-compliant balloon with good angiographic and tomographic (OCT) results. A translesional pressure gradient was not measured. Blood pressure normalized immediately after the procedure, and she was discharged on $20 \mathrm{mg}$ telmisartan because of left ventricular dysfunction/hypertrophy and aspirin $75 \mathrm{mg}$ daily. After one year of follow-up, she gradually started having a recurrence of headache and dyspnea. Home measurements revealed multiple blood pressure recordings of $>180 / 90$ requiring escalation of anti-hypertensives. A repeat renal Doppler was suggestive of bilateral renal artery stenosis, and a renal artery angiography revealed $90 \%$ bilateral focal restenosis at the previous sites (Figure 2A and B). OCT of the left renal artery revealed focal stenosis with homogeneously thickened media with fibrosis in the inner half, as on the initial imaging (Figure 2C-F). No dissection flap was seen this time. The distal reference vessel was $4.2 \mathrm{~mm}$. OCT of the right renal artery was suggestive of medial fibroplasia as before, with the distal reference diameter of $4.0 \mathrm{~mm}$.

Subsequently, after initial balloon angioplasty with a $4.0 \mathrm{~mm}$ and then a $4.5 \times 12 \mathrm{~mm}$ balloon, a pressure gradient of $15 \mathrm{mmHg}$ was noticed across the lesion in the left 


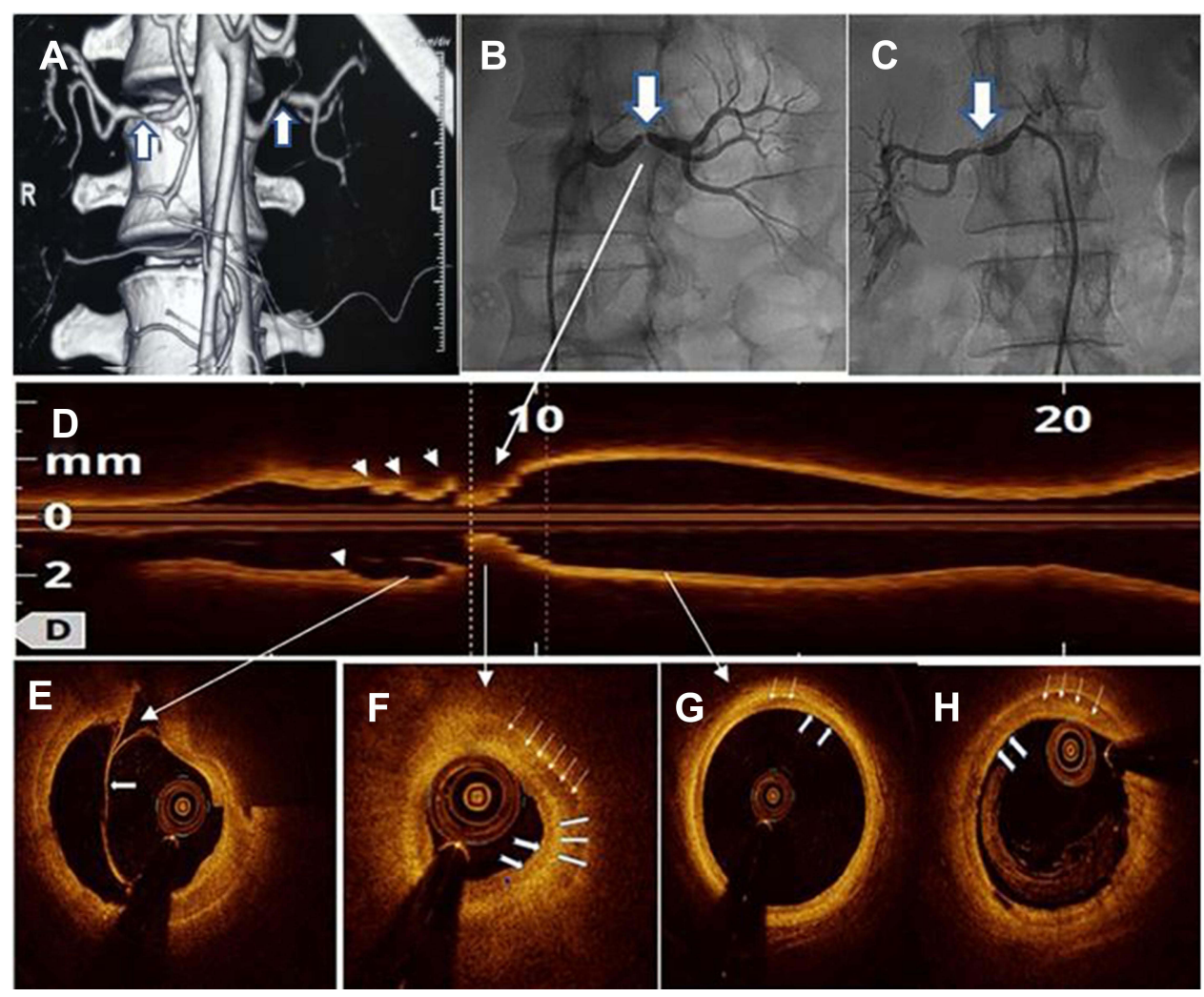

Figure I Various Imaging modalities used in diagnosing Renal artery stenosis in fibromuscular dysplasia.

Notes: (A) CT angiography shows bilateral critical stenosis of renal arteries at mid-segment (arrows). (B and C) Selective renal angiography shows bilateral mid-segment focal stenosis of renal arteries (arrows). OCT imaging shows (D) longitudinal reconstruction with a string of beads appearance (arrowheads). (E) An intima-medial dissection by an intimal flap (arrow), (F) thickened media (six-line arrows) along with fibrosis in the inner half (three-block arrows), characteristic of predominantly medial fibroplasia and normal intima (two notched arrows). (G) Normal Intima and Media (arrows) in reference segment. (H) Post-angioplasty right renal artery OCT shows medial fibroplasia (line arrows) with normal intima (notched arrows).

renal artery. Further dilation with a $5 \times 10 \mathrm{~mm}$ balloon followed by stenting with a $5 \mathrm{~mm} \times 15 \mathrm{~mm}$ renal stent system (RX Herculink Elite stent, Abbott Vascular, Abbott Park, IL) lead to resolution of pressure gradient across the lesion on the left side. The decision to stent the lesion was taken due to the presence of restenosis and residual gradient. Angiographic and OCT imaging confirmed good stent expansion and apposition (Figure 3A-E). On the right side, there was no pressure gradient after balloon angioplasty with a $4.5 \mathrm{~mm}$ balloon, so stenting was not performed (Figure $3 \mathrm{C}$ and $\mathrm{F}$ ). At six months follow-up after the reintervention, she remains normotensive without any medications. Currently, she is on aspirin $75 \mathrm{mg}$ daily.

\section{Discussion}

FMD is a rare, idiopathic, non-inflammatory, nonatherosclerotic condition, which usually involves small and medium-sized arteries. Renal artery involvement is common, frequently bilateral, usually presents with hypertension, and significantly increases the risk of preeclampsia in women of childbearing age. ${ }^{7}$ Originally classified histologically by Harrison \& McCormack based on the layer of the artery predominately involved, ${ }^{8}$ medial fibroplasia and peri-medial fibroplasia with characteristics string of beads appearance account for is $75-80 \%$ and $10-$ $15 \%$ of cases whereas medial hyperplasia and intimal fibroplasia with angiographic focal, concentric stenosis, account for $1-2 \%$, and $<10 \%$ of cases respectively. Adventitial type is the least common $(<1 \%)$ lesion with sharply localized, tubular areas of stenosis. ${ }^{9}$ In the current era of endovascular therapy, surgical specimens are rarely available for histological examination. So FMD was later adapted to angiographic classification through histological-angiographic correlation. ${ }^{1}$ American Heart Association consensus document (2014) classified FMD angiographically into focal and multifocal types. Multifocal FMD with a 'string of beads' appearance is the most common type (80-90\%), usually involves mid and distal portions of the artery, and is represented by medial fibroplasia virtually in all adults. On the other hand, focal FMD may occur in any 


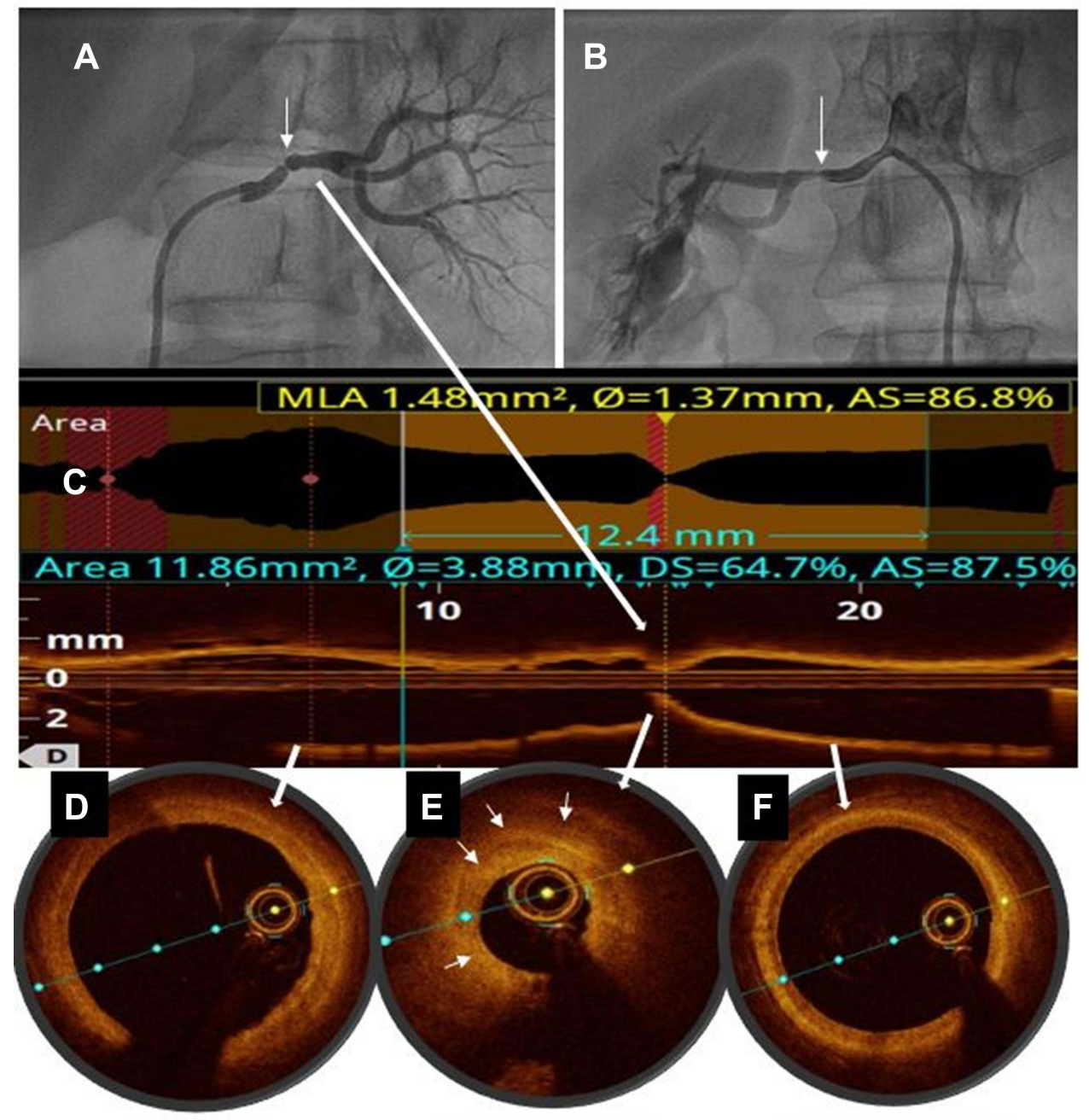

Figure 2 Current bilateral renal angiogram showing significant restenosis (arrows) in the left (A) and right (B) renal arteries. Longitudinal reconstruction of optical coherence tomography (OCT) imaging (C) of left renal artery depicting the corresponding lesion indicated by white arrows. Cross-sectional images showing distal (D) and proximal $(\mathbf{F})$ reference zones are free of disease and measure $4.2 \mathrm{~mm}$. The area of focal restenosis on the angiogram correlate with extensive medial and intimal hyperplasia (arrows) on OCT (E), causing $87 \%$ area (re) stenosis.

part of the renal artery and is usually caused by intimal fibroplasia or sometimes by medial hyperplasia or adventitial type. ${ }^{3}$ The majority of patients with a multifocal variant have a typical presentation of renal FMD as a middle-aged woman with hypertension, whereas focal FMD patients are usually $<30$ years with an average BP higher than the multifocal variant. ${ }^{10}$

Catheter-based angiography, a gold standard for diagnosing $\mathrm{FMD},{ }^{7}$ is superior to other imaging modalities and offers an opportunity for endovascular therapy. ${ }^{11}$ Albeit angiographic multifocal stenosis is typical of FMD, yet focal stenosis requires further evaluation. In such a situation, OCT imaging appears to be more promising for diagnosing and classifying FMD, at least in a mediumsized renal artery. As FMD commonly involves intima and media, high resolution of OCT allows easy assessment of morphology, vascular characteristics, and differentiation from other causes of RAS. OCT imaging in our case reclassified angiographically focal lesion as medial fibroplasia with a characteristic beaded appearance of multifocal disease, which was not discernible on conventional angiography.

Limited data focusing on the systematic role of OCT in the diagnosis and classification of FMD is available. In addition to illustrating the benefits of OCT in diagnosis and intervention in RAS in FMD as reported by Mizutani et $\mathrm{al}^{2}{ }^{2}$ our case additionally explores the utility of OCT in correctly classifying the type of FMD in the angiographically ambiguous lesion. Proper classification of FMD could have prognostic implications as hypertension cure 


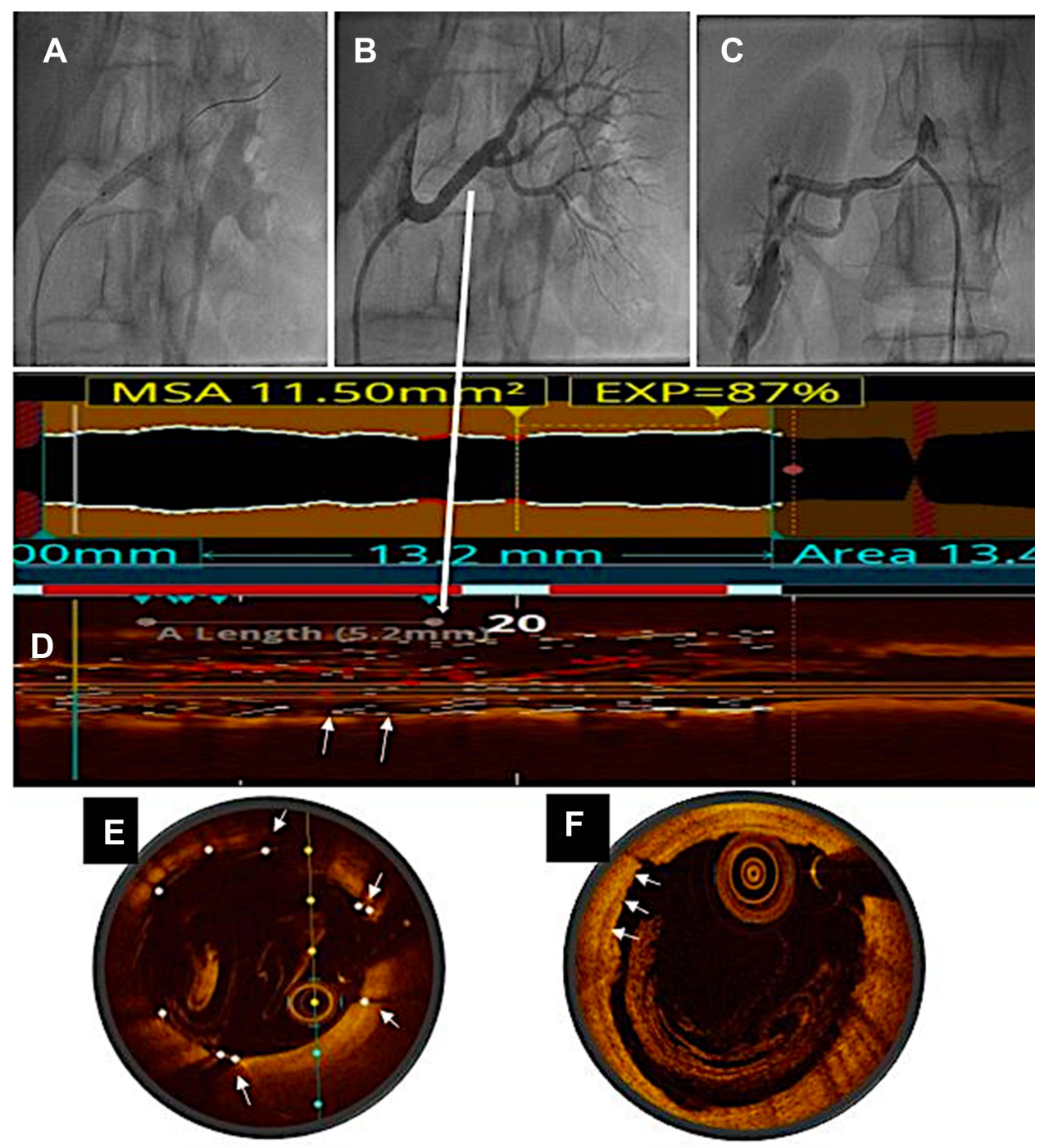

Figure 3 Angiogram showing stent deployment in the left renal artery (A) with an excellent angiographic result (B). The right renal artery shows (C) a good result with balloon angioplasty alone. Longitudinal (D) and cross-sectional (E) OCT images of left renal artery show good stent expansion and apposition (arrows) without residual stenosis. Cross-sectional OCT image (F) of right renal artery after balloon angioplasty shows good result with some intimal tears (arrows) and no residual stenosis (correlate with Image (C)).

rate after interventions could be better in focal versus the multifocal type of FMD. ${ }^{12}$ Accordingly, we contemplate the characterization with OCT in guiding the classification of FMD, especially in angiographic focal or ambiguous disease. However, as the existing literature on the use of OCT to diagnose renal FMD is limited to a few case reports, the diagnostic accuracy of OCT for renal FMD and its ability to differentiate from atherosclerosis needs to be demonstrated in larger studies. Also, the correlation between OCT findings and histology in the diagnosis of FMD requires validation.

Revascularization is indicated in patients with resistant hypertension, intolerance or non-compliance to medication, and progressive renal dysfunction. Over the years, endovascular intervention has replaced traditional surgical repair as the first-line therapy. ${ }^{1,3}$ In general, balloon angioplasty is the treatment of choice. Stenting is reserved for lesions with suboptimal results after balloon angioplasty. However, high recurrence rates of around $28 \%$ at five years have been reported. ${ }^{6}$ As a result, many have advocated an upfront stenting strategy to avoid restenosis and repeat interventions. ${ }^{13,14}$ Stenting is best avoided in distal renal arteries and smaller renal arteries because of the higher restenosis rate. ${ }^{3,4,6}$ For balloon angioplasty, the semi-compliant balloon is sized $1: 1$ to the reference vessel diameter. The non-compliant or cutting balloon is usually avoided for the risk of rupture. Despite good angiographic appearance after balloon 
angioplasty, the results may be suboptimal as in our case. The usual approach in such a case is to gradually increase the balloon size until the translesional gradient resolves. Nevertheless, it carries the risk of vascular injuries like rupture or dissection, ${ }^{3}$ significantly if device size far exceeds the vessel size. Measuring the vessel size with intravascular imaging like OCT may guide correct selection of balloon/stent size. In the index case, though the vessel was $4.1 \mathrm{~mm}$, we could confidently use a $5 \mathrm{~mm}$ stent with OCT guidance, which resolved the translesional gradient with minimal risk of any complication. However, angiographic appearance alone should not be used as a surrogate for successful intervention. Measurement of the pressure gradient is also essential to confirm the procedural success. ${ }^{15}$ The persistent residual gradient may be due to inadequate lesion dilatation, recoil, or a dissection flap which may require a stent. In the index case, we had an excellent angiographic and OCT outcome during the first intervention; however, the translesional gradient was not measured. During the subsequent intervention for restenosis, a persistent residual pressure gradient despite good angiographic results after balloon angioplasty required stenting. For this, OCT guidance was indispensable in choosing the stent size and achieving a complete resolution of the translesional pressure gradient.

Due to its higher resolution than intravascular ultrasound (IVUS), OCT is ideally suited for assessing the intima and media of the vessels. ${ }^{16}$ However, it has poor penetration limits visualization of distant structures. ${ }^{17}$ Another problem is the difficulty in obtaining clearance of blood for OCT imaging in large arteries. In the index case, although with difficulty, we were able to achieve good blood clearance and OCT images of FMD of the renal arteries.

In conclusion, virtual histology on OCT imaging aids in confirming the diagnosis in angiographically ambiguous focal disease. Further, in addition to understanding the mechanism of restenosis, OCT imaging may add safety in selecting balloon/stent size and optimize the intervention. Good angiographic results should always be supplemented with pressure gradient measurement to confirm technical success. Residual gradient after balloon angioplasty may require stenting, especially in the case of restenosis. Further research is warranted to know additional factors that predispose to restenosis, and an upfront stenting strategy may be beneficial in such circumstances.

\section{Data Sharing Statement}

The datasets used and analyzed during the case report are available from the corresponding author on reasonable request.

\section{Ethics Approval and Consent to Participate}

Published research was conducted ethically in accordance with the Declaration of Helsinki. Institutional approval was not required to publish the case report.

\section{Consent for Publication}

The patient gave her written informed consent to publish the case.

\section{Acknowledgments}

The authors acknowledge the technical help provided by Ramneek Atreya in preparing the OCT images.

\section{Disclosure}

The authors report no conflicts of interest in this work.

\section{References}

1. Gornik HL, Persu A, Adlam D, et al. First international consensus on the diagnosis and management of fibromuscular dysplasia. Vasc Med. 2019;24(2):164-189. doi:10.1177/1358863X18821816

2. Mizutani K, Itoh A, Sugioka K, Komatsu R, Naruko T, Yoshiyama M. Intravascular findings of fibromuscular dysplasia on optical coherence tomography. J Cardiol Cases. 2015;12(2):39-42. doi:10.1016/j. jecase.2015.03.009

3. Olin JW, Gornik HL, Bacharach JM, et al. Fibromuscular dysplasia: state of the science and critical unanswered questions: a scientific statement from the American Heart Association. Circulation. 2014;129(9):1048-1078. doi:10.1161/01.cir.0000442577.96802.8c

4. Gottsäter A, Lindblad B. Optimal management of renal artery fibromuscular dysplasia. Ther Clin Risk Manag. 2014;10:583. doi:10.2147/ TCRM.S48746

5. Trinquart L, Mounier-Vehier C, Sapoval M, Gagnon N, Plouin P-F. Efficacy of revascularization for renal artery stenosis caused by fibromuscular dysplasia: a systematic review and meta-analysis. Hypertension. 2010;56(3):525-532. doi:10.1161/HYPERTENSIONAHA.110.152918

6. Davies MG, Saad WE, Peden EK, Mohiuddin IT, Naoum JJ, Lumsden AB. The long-term outcomes of percutaneous therapy for renal artery fibromuscular dysplasia. J Vasc Surg. 2008;48(4):865-871. doi:10.1016/j.jvs.2008.05.030

7. Vance CJ, Taylor RN, Craven TE, Edwards MS, Corriere MA. Increased prevalence of preeclampsia among women undergoing procedural intervention for renal artery fibromuscular dysplasia. Ann Vasc Surg. 2015;29 (6):1105-1110. doi:10.1016/j.avsg.2015.03.037

8. Harrison EG, McCormack LJ. Pathologic classification of renal arterial disease in renovascular hypertension. Mayo Clin Proc. 1971;46 (3):161-167.

9. McCormack LJ, Poutasse EF, Meaney TF, Noto TJ Jr, Dustan HP. A pathologic-arteriographic correlation of renal arterial disease. $A m$ Heart J. 1966;72(2):188-198. doi:10.1016/0002-8703(66)90442-X 
10. Kincaid OW, Davis GD, Hallermann FJ, Hunt JC. Fibromuscular dysplasia of the renal arteries: arteriographic features, classification, and observations on natural history of the disease. Am J Roentgenol. 1968;104(2):271-282. doi:10.2214/ajr.104.2.271

11. Olin JW, Pierce M. Contemporary management of fibromuscular dysplasia. Curr Opin Cardiol. 2008;23(6):527-536. doi:10.1097/ HCO.0b013e328313119a

12. Savard S, Steichen O, Azarine A, Azizi M, Jeunemaitre X, Plouin P-F. Association between 2 angiographic subtypes of renal artery fibromuscular dysplasia and clinical characteristics. Circulation. 2012;126(25):3062-3069. doi:10.1161/CIRCULATIO NAHA.112.117499

13. Mann SJ, Sos TA. The cardiovascular outcomes in renal atherosclerotic lesions study and the future of renal artery stenting. $J$ Clin Hypertens. 2014;16(3):162-165. doi:10.1111/jch.12270
14. Edem E, Aksoy MN, Pabuccu MT, Tatlı E. Endovascular treatment of renal artery stenosis due to fibromuscular dysplasia-is stent implantation underused in this circumstance? Heart Views. 2016;17(2):69.

15. Olin JW, Sealove BA. Diagnosis, management, and future developments of fibromuscular dysplasia. J Vasc Surg. 2011;53(3):826-836. doi:10.1016/j.jvs.2010.10.066

16. Kume T, Akasaka T, Kawamoto T, et al. Assessment of coronary intima-media thickness by optical coherence tomography comparison with intravascular ultrasound. Circ J. 2005;69(8):903-907. doi:10.1253/circj.69.903

17. Hao H, Fujii K, Shibuya M, et al. Different findings in a calcified nodule between histology and intravascular imaging such as intravascular ultrasound, optical coherence tomography, and coronary angioscopy. JACC Cardiovasc Interv. 2014;7(8):937-938. doi:10.1016/j.jcin.2013.12.212

\section{Publish your work in this journal}

The International Medical Case Reports Journal is an international, peer-reviewed open-access journal publishing original case reports from all medical specialties. Previously unpublished medical posters are also accepted relating to any area of clinical or preclinical science. Submissions should not normally exceed 2,000 words or 4 published pages including figures, diagrams and references. The manuscript management system is completely online and includes a very quick and fair peer-review system, which is all easy to use. Visit http://www.dovepress.com/testimonials.php to read real quotes from published authors. 